\title{
Magnifying miniature displays with microlenslet arrays
}

\author{
Vesselin Shaoulov*, Ricardo Martins and Jannick Rolland \\ CREOL / School of Optics \\ University of Central Florida \\ Orlando, Florida 32816 \\ *Email:vesko@odalab.ucf.edu
}

\begin{abstract}
Current technology trends are focused on miniaturizing displays, although for specific applications such as the use of head-mounted displays (HMD) this limits the advancements for a wider field-of-view (FOV) and a negligible overall weight of the optics. Due to the advancements of electronics that benefit from smaller miniature displays, universities and companies are focused on developing this technology to meet the growing demand of this global market. Higher resolution displays with added brightness are being developed, but these displays are decreasing in their viewable area. HMDs can benefit from these higher resolution and brighter displays but they will undergo an increased optical weight to compensate for the smaller display size. To overcome this hindrance in HMDs, we demonstrate in this paper how to incorporate microlenslet arrays as an optical relay system to magnify miniature displays. Microlenslet arrays provide respectively shorter focal length which yields a smaller overall object to image distance and an incremental overall weight compared to an otherwise increased optical lens assembly. The contribution of this paper is a patented concept of magnifying/demagnifying miniature displays with microlenslet arrays that can be integrated in a spaced limited area.
\end{abstract}

\section{INTRODUCTION}

Many applications require the integration of an ultra-compact optical system to magnify miniature displays. For example, a driving application for our research comprises of the magnification of miniature displays in head-mounted displays (HMDs). ${ }^{1}$ A key component of any HMD is the microdisplay, specifically its size, resolution, and illumination scheme, which drive the design, and thus the final layout after packaging. Several technologies, such as liquid crystal displays (LCD), organic light emitting diodes (OLED) and liquid crystal on silicon displays (LCOS) currently compete for the microdisplay market. ${ }^{2,3,4}$ They all have specific advantages and are best fit for different design requirements and applications. 


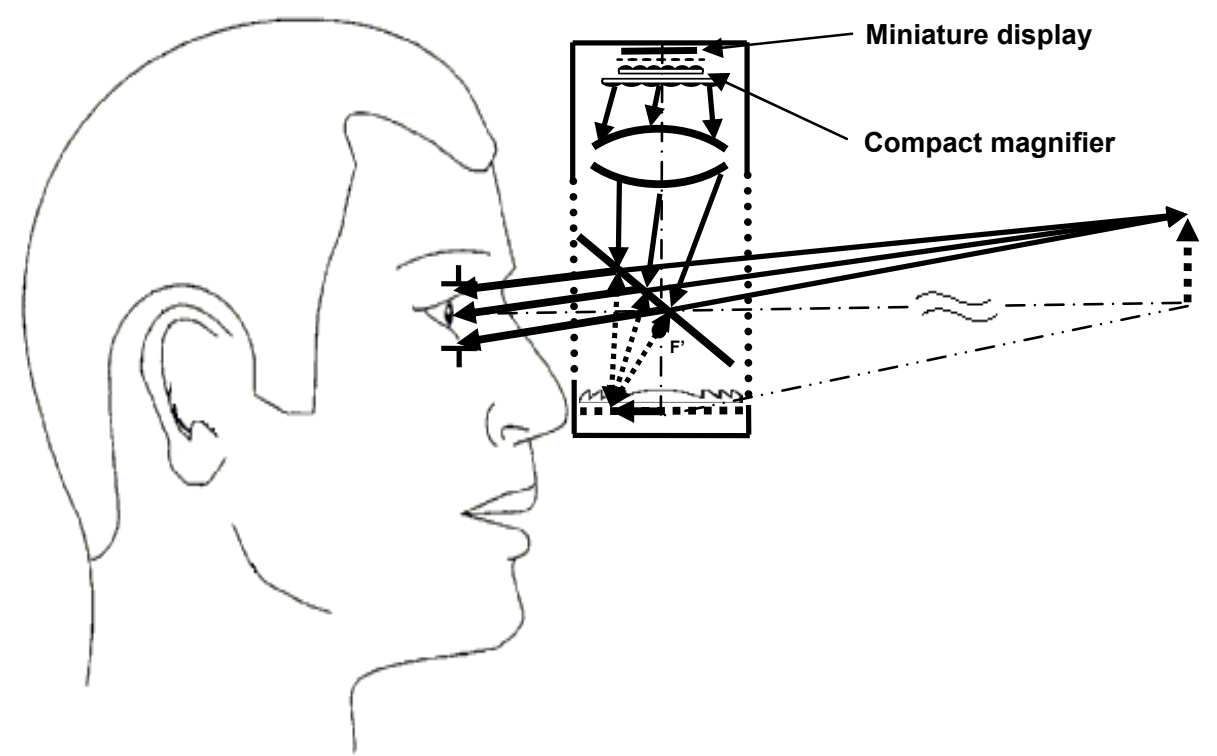

Fig. 1. Conceptual design of an HMD with integrated miniature display, magnified by a compact magnifier.

An example of an integrated system comprising of a miniature display magnified by a compact magnifier is presented in Fig. 1 for a recently conceived HMD. ${ }^{5}$ In such configuration, magnification of the miniature display is needed to minimize the overall length of the optical assembly to further increase the field of view (FOV) of the HMD. As part of our previous work, we designed a state-of-the-art 1:2 magnifying system utilizing bulk macro optics, however while compact, this system still had an overall object to image length (OAL) of $120 \mathrm{~mm}$ and weighted over 700 grams. $^{6}$ Therefore an alternative approach had to be investigated. Given the compactness requirements imposed on any HMD system, a magnifying system with size of a few millimeters and weight of a few grams is desired. Optical magnifying systems based on microlenslet arrays could provide a useful solution for such applications. The key contribution of our investigation is the replacement of bulk macro optics with multi-aperture micro-optics.

\section{KEY CHALLENGES IN IMAGING WITH MICROLENSLET ARRAYS}

The basic theory of imaging with microlenslet arrays, developed by R.H. Anderson, was driven by requirements of optical scanning devices. ${ }^{7}$ In his work, Anderson demonstrated that arrays of simple lenses combined with appropriate baffles could be used in close-up imaging systems for black and white document copiers, oscilloscope cameras, as well as binary code scanners. Microlenslet-array based imaging systems were consequently further investigated for optical scanners and copiers, ${ }^{8,9}$ and $3 \mathrm{D}$ integral photography. ${ }^{10}$ 
The imaging capabilities of microlenslet arrays for either grayscale or color images were previously investigated, and it was demonstrated that 1:1 compact relays for such images could be conceived with OAL of less than $7 \mathrm{~mm}^{11}$

The two key challenges when imaging with multi-aperture stacks of microlenslet arrays are the formation of ghost images in the system and lensletization. ${ }^{11}$ The formation of ghost images in a multi-aperture imaging system comprising of a stack of microlenslet arrays is caused by the light emitted from the object in all directions taking more than one optical path through the system. The formation of ghost images in such optical system is demonstrated in Fig. 2(a). To suppress the ghost image formation an appropriate baffle should be placed in the location of either the entrance or the exit pupil of the optical system as demonstrated in Fig. 2(b).

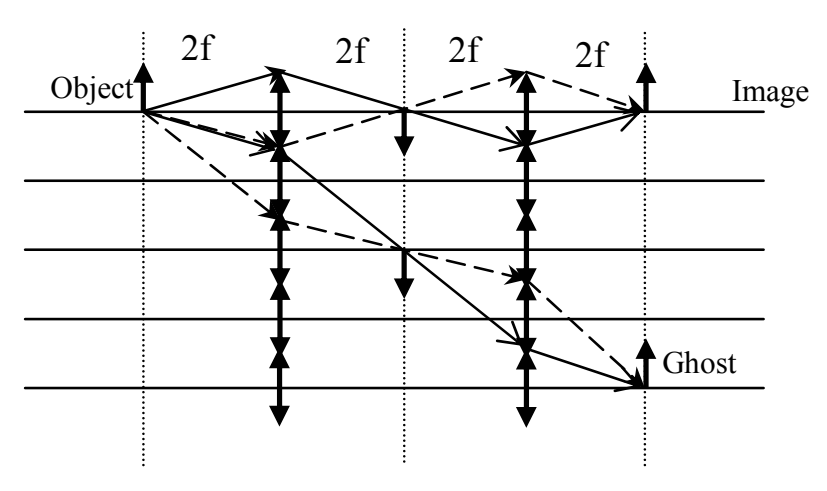

(a)

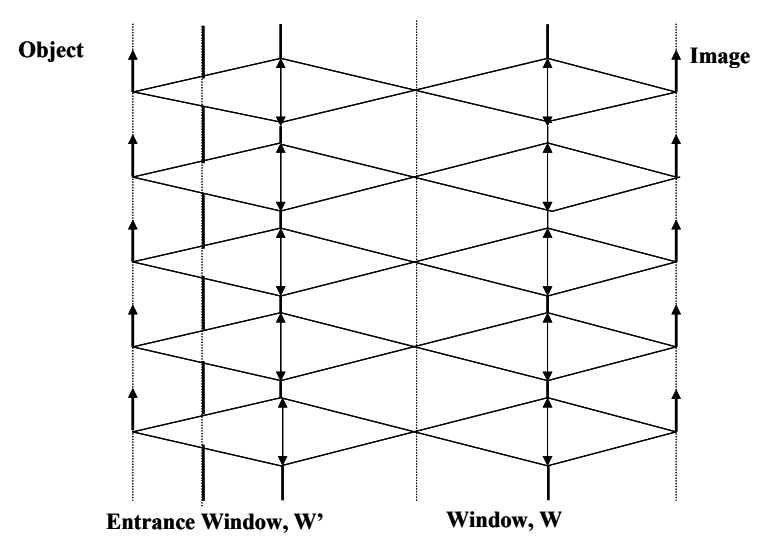

(b)

Fig. 2. First order optical layout of a compact imaging system, consisting of a multi-aperture stack of microlenslet arrays, demonstrating (a) the formation of ghost images in the system without an appropriate baffle, and (b) the suppression of ghost images formation with an appropriate baffle placed in the location of the entrance pupil.

The lensletization is another property of optical imaging with a multi-aperture stack of two microlenslet arrays that describes the sampling of the object by each pair of microlenslets in the stacks, where each pair operates over a limited field of view. Overcoming this effect is less straightforward than suppressing ghost images and requires overlapping the individual sub-fields of view of each individual pair of lenses, as demonstrated in Fig. 3(a) and 3(b). The overlap of the individual sub-fields of view occurs at the expense of an increase in the OAL and a natural decrease in resolution. ${ }^{11}$ 


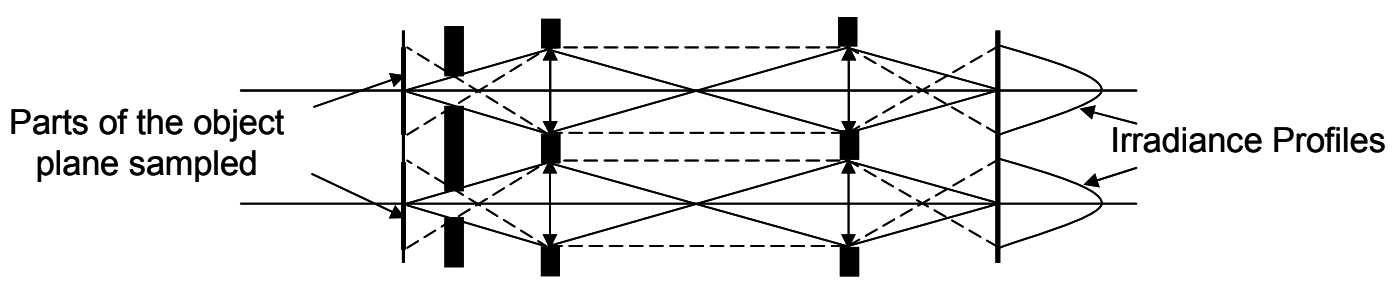

(a)

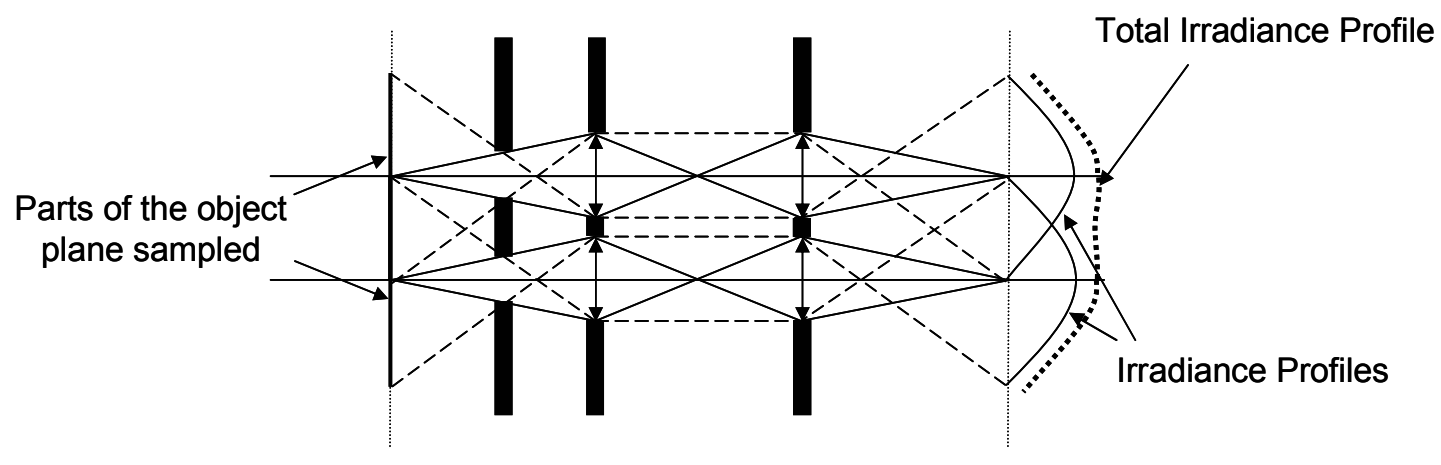

(b)

Fig. 3. First order optical layout of a compact imaging system, consisting of a multi-aperture stack of microlenslet arrays, demonstrating the lensletization effect (a) without overlapping the individual sub-FOVs leading to sampling of the object by each individual pair of lenses in the stack, and (b) with overlapping the individual sub-FOVs suppressing the lensletization effect and achieving an uniform image.

\section{OPTICAL LAYOUT OF MAGNYFYING MINIATURE DISPLAYS WITH A STACK OF TWO MICROLENSLET ARRAYS}

The concept of imaging with a multi-aperture stack of two microlenslet arrays was further extended to the most general case, where two dissimilar arrays were used to achieve a system with overall magnification or demagnification, as demonstrated in Fig 4.

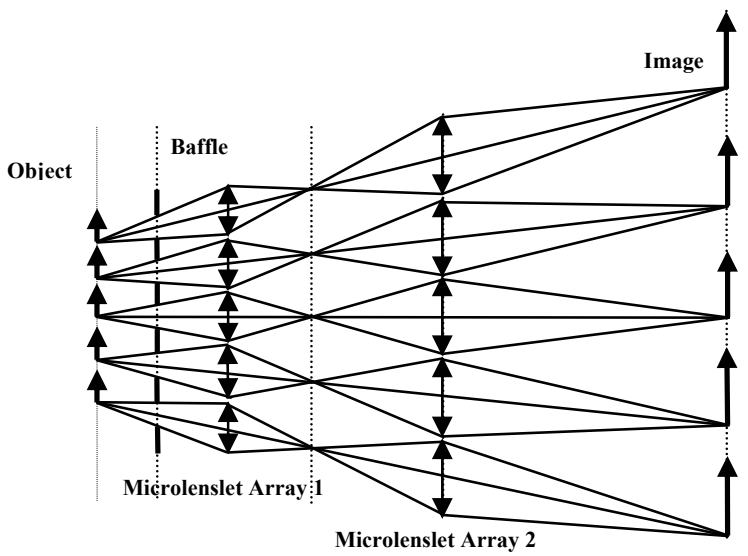

Fig. 4. First order optical layout of compact magnifying system consisting of a multi-aperture stack of microlenslet arrays. 
In this configuration the first and the second microlenslet arrays are of focal lengths $f_{l}$ and $f_{2}$, diameters $D_{l}$ and $D_{2}$, and operate at magnifications $m_{l}$ and $m_{2}$, respectively. The overall magnification of such system is $M=m_{1} m_{2}$. An appropriate baffle is placed in the location of the entrance pupil of the system to suppress the ghost image formation and the system is configured with enough overlap to minimize the lensletization. The optical and the geometrical relationships used to achieve such configuration were discussed previously. ${ }^{12,13}$

\section{THEORETICAL MODELING}

In order to further analyze the imaging properties of microlenslet array based magnifiers, a computer model was developed using custom-designed software based on ASAP ${ }^{\mathrm{TM}}$. The first aspect of modeling is to define an appropriate light source or equivalently an object to be imaged. Since the driving application for our research was to magnify miniature displays, a complex grayscale light source, such as a bitmap portrait, was selected to assess the grayscale imaging capability of the proposed microlenslet array based magnifier. In the case of grayscale images, image quality may be assessed subjectively as well as with more sophisticated quantitative approaches. ${ }^{11,12}$

The optical layout of a 1:2 system using two arrays of each 11 by 11 microlenses, combined with an associated baffle located at the entrance pupil of the system is shown in Fig 5.

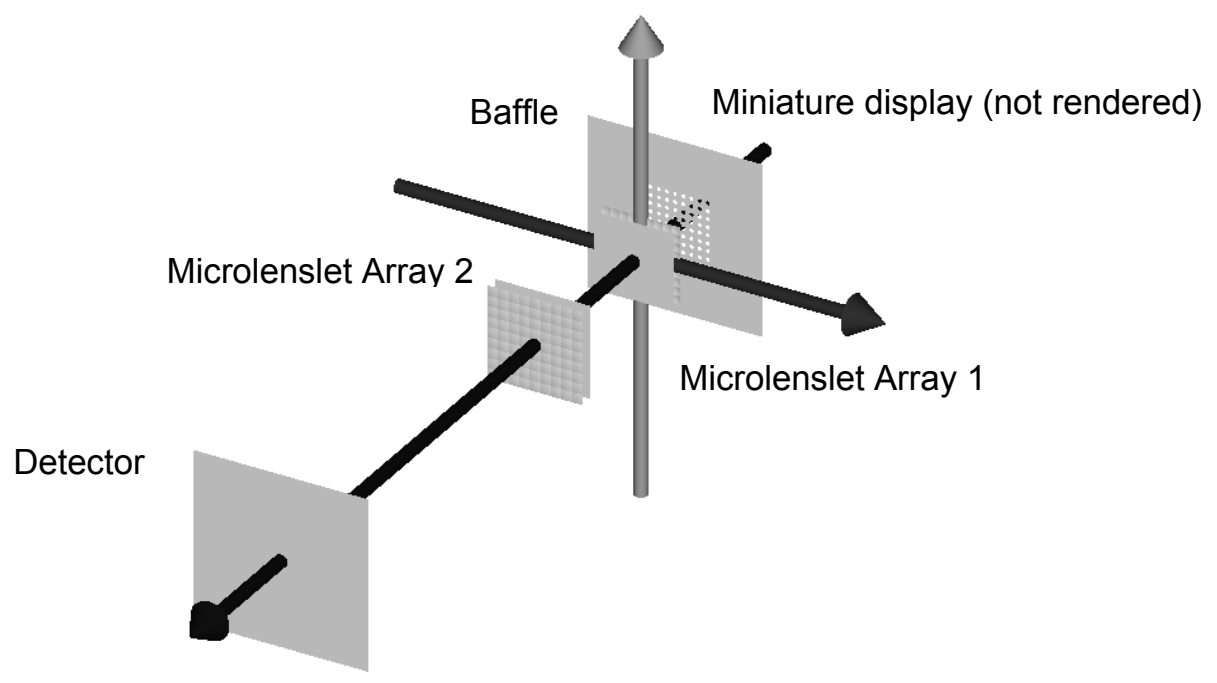

Fig. 5. $\mathrm{ASAP}^{\mathrm{TM}}$ model of a compact magnifying system consisting of a multi-aperture stack of microlenslet arrays. 
Each lens in the first array was considered an F/5 square plano-convex, of $150 \mu \mathrm{m}$ thickness, and $500 \mu \mathrm{m}$ focal length. Each lens in the second array was an F/8.3 square plano-convex lens, of $150 \mu \mathrm{m}$ thickness, and $1000 \mu \mathrm{m}$ focal length. Because we use simple plano-convex singlets, which inherently have significant axial chromatic aberration, we only consider imaging single color grayscale image, which we selected without loss of generality to be $\lambda$ equal $656 \mathrm{~nm}$. In our previous work we demonstrated that one level of optimization in optical raytracing is to direct the rays towards the entrance pupil of the optical system. ${ }^{11}$ While in the case of microlenslet arrays no single pupil exists but instead multi-sub-pupils must be considered, a fictitious pupil is defined that encompasses all the sub-pupils. ${ }^{11}$ Building on this scattering technique, which is standard in $\mathrm{ASAP}^{\mathrm{TM}}$ software, the raytrace was further optimized. The rays were first traced from the source to the diffuser, and then only the scattered rays were traced from the diffuser towards the fictitious entrance pupil of the system. An analysis of the minimum number of rays to achieve $97 \%$ accuracy, which is enough for a first order feasibility assessment, demonstrated that a total of 150 million rays should be traced through the system. ${ }^{14}$

Results of feasibility simulations using a grayscale light source and 150 million rays are shown in Fig. 5 for the imaging configuration shown in Fig. 6.

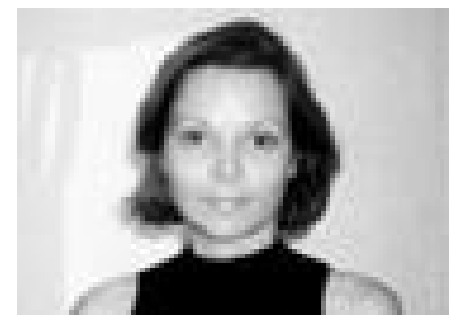

(a)



(b)

Fig. 6. Simulation of imaging of a grayscale object projected by a miniature display through a stack of two dissimilar microlenslet arrays: (a) a grayscale object and (b) magnified image through the system. 


\section{CONCLUSION AND FUTURE WORK}

Results presented in this paper demonstrate that ultra-compact multi-aperture magnification system based on a stack of two dissimilar microlenslet arrays is feasible since a final image with no apparent ghosting and a small residual lensletization can be formed. That will allow for efficient magnification of miniature displays in an HMD. As part of the future work, we will investigate the higher order image properties of such systems and will optimize for best performance to satisfy a spatial frequency of approximately 24 cycles $/ \mathrm{mm}$ to drive 0.6 inch 800x600 miniature displays.

\section{ACKNOWLEDGMENTS}

We thank BRO Corporation for the educational license of ASAP ${ }^{\mathrm{TM}}$ to the School of Optics/CREOL/FPCE. This research was supported in part by the US Army Simulation, Training, and Instrumentation Command (STRICOM) and funded in part by grant number N00014-03-10677 awarded by the Office of Naval Research.

\section{REFERENCES}

1. J. P. Rolland, H. Fuchs, "Optical versus Video See-Through Head-Mounted Displays", in "Fundamentals of wearable computers and augmented reality", W. Barfield, T. Caudell, Lawrence Erlbaum Associates, New Jersey 2001.

2. W.C. O'Mara, C. William, "Liquid Crystal Flat Panel Displays: Manufacturing Science \& Technology" Van Nostrand Reinhold, New York, 1993.

3. M. E. Thompson, S. R. Forrest, P. Burrows, Z. Shen, G. Gu and V. Bulovic, "The stacked OLED (SOLED), a new type of organic device for achieving high-resolution full-color displays", Synthetic Metals Vol. 91(1-3), p. 9-13, December 1997.

4. Intel(R) LCOS Technology Brief, ftp://download.intel.com/design/celect/technology/lcos/30042801.pdf, 2004.

5. R. Martins and J. Rolland, "Diffraction of Phase Conjugate Material in a New HMD Architecture", SPIE Proceedings Vol. 5186, p. 277-283, SPIE AeroSense: Helmet and Head-Mounted Displays VIII: Technologies and Applications, Editors: C. E. Rash and C. E. Reese, September 2003. 
6. J.P. Rolland, H. Hua, and V. Shaoulov, "Design of a compact relay lens", Technical Report TR0205, University of Central Florida 2002.

7. R.H. Anderson, "Close-up imaging of documents and displays with lens arrays", Applied Optics Vol. 18(4), p. 477-484, February 1979.

8. J. Mir, "High resolution optical-addressing device and electronic scanner and/or printer apparatus employing such device”, US Patent 4,377,753, 22 March 1983.

9. M. Kawazi and Y. Ogura, "Application of gradient index fiber arrays to copying machines", Applied Optics 19(7), p. 1105-1112, April 1980

10. L. Erdmann, K. J. Gabriel, "High-Resolution Digital Integral Photography by use of a Scanning Microlens Array", Applied Optics 40(31), p. 5592-5599, November 2001.

11. V. Shaoulov, J. Rolland, "Design and Assessment of Microlenslet-Array Relay Optics", Applied Optics 42(34), p. 6838-6845, December 2003.

12. V. Shaoulov, R. Martins, J. Rolland, “Compact microlenslet array-based magnifier”, Optics Letters 29(7), p. 709-711, April 2004.

13. V. Shaoulov, R. Martins and J. Rolland, "Imaging with microlenslet arrays", Annual Meeting of SPIE 2003, SPIE Proceedings Vol. 5174, p. 11-18, Novel Optical Systems Design and Optimization VI; Eds. Jose M. Sasian, R. John Koshel, Paul K. Manhart, November 2003.

14. W. Cassarly, "Art of making efficient illuminator design fun”, Annual Meeting of SPIE 2003, SPIE Proceedings Vol. 5186, p. 1-4, Design of Efficient Illumination Systems, Editor: R. John Koshel, November 2003. 